\title{
Impact of Serum Biomarkers and Clinical Factors on Intensive Care Unit Mortality and 6-Month Outcome in Relatively Healthy Patients with Severe Pneumonia and Acute Respiratory Distress Syndrome
}

\author{
Chia-Cheng Tseng, 1,2 Wen-Feng Fang, 1,2,3 Sum-Yee Leung, ${ }^{1,2}$ Hung-Chen Chen, ${ }^{1}$ \\ Ya-Chun Chang, ${ }^{1}$ Chin-Chou Wang, ${ }^{1,2,3}$ Huang-Chih Chang, ${ }^{1,2}$ and Meng-Chih Lin ${ }^{1,2,3}$ \\ ${ }^{1}$ Division of Pulmonary and Critical Care Medicine, Department of Internal Medicine, \\ Kaohsiung Chang Gung Memorial Hospital and Chang Gung University College of Medicine, Kaohsiung 83301, Taiwan \\ ${ }^{2}$ Department of Respiratory Care, Chang Gung University of Science and Technology, Chiayi 813, Taiwan \\ ${ }^{3}$ Department of Respiratory Therapy, Kaohsiung Chang Gung Memorial Hospital and Chang Gung University College of Medicine, \\ Kaohsiung 83301, Taiwan
}

Correspondence should be addressed to Meng-Chih Lin; linmengchih@hotmail.com

Received 30 December 2013; Accepted 29 January 2014; Published 3 March 2014

Academic Editor: Vincent Sapin

Copyright (C) 2014 Chia-Cheng Tseng et al. This is an open access article distributed under the Creative Commons Attribution License, which permits unrestricted use, distribution, and reproduction in any medium, provided the original work is properly cited.

\begin{abstract}
Objectives. This study aimed to identify the independent biomarkers and clinical factors that could predict ICU mortality and 6month outcomes in relatively healthy patients with severe pneumonia and acute respiratory distress syndrome (ARDS). Patients and Methods. We prospectively enrolled patients with severe pneumonia-related ARDS that required mechanical ventilation. Patients were excluded if they were unable to take care of themselves. Several biomarkers and clinical factors were evaluated prospectively on day 1 and day 3 after ICU admission. All biomarkers and clinical factors were collected for analysis. Results. 56 patients were enrolled in this study. We determined that the initial appropriate antibiotics use was an independent clinical factor and day 1 high-mobility group protein B1 (HMGB1) concentration was an independent biomarker for ICU mortality. Interestingly, we also found that a low day 1 albumin level was an independent biomarker for predicting patient life dependence 6 months after a pneumonia event. Conclusion. Patients with severe pneumonia and ARDS requiring mechanical ventilation experience high rates of ICU mortality or disability, even if they were quite healthy before. Initial appropriate antibiotics use and day 1 level of HMGB1 were independent factors for predicting ICU mortality. Day 1 albumin level was predictive of 6-month patient life dependence.
\end{abstract}

\section{Introduction}

Pneumonia is associated with a high morbidity and mortality rate throughout the world [1]. Acute lung injury (ALI) and acute respiratory distress syndrome (ARDS) are important denouements of severe pneumonia and are associated with high mortality rates $[2,3]$ despite recent improvement in prognosis [4-6]. Sepsis (both from pulmonary and extrapulmonary origin) is a leading etiology of ALI/ARDS, and lung infection may account for up to $50 \%$ of ARDS cases $[3,7]$.
Although patients with severe pneumonia and ARDS can survive, survivors also frequently experience major longterm morbidities that impair their pulmonary, neuromuscular, physical, cognitive, and psychological function. In turn, these impairments affect the survivors' overall quality of life (QOL) $[8,9]$. Previous studies also suggested that longterm survival is unaffected by ARDS status; however, ARDS severely affects QOL, functional independence, and cognitive function [10-12]. In relatively healthy patients bearing few underlying diseases, severe pneumonia with ARDS status 
rarely occurs; however, patients that are relatively young and healthy when they develop ARDS may never recover completely and may experience ongoing functional limitations after an episode of critical illness [13]. This may be attributed to persistent intensive care unit- (ICU-) acquired weakness, in addition to a variety of other physical and mental health impairments [14]. To date, few studies have been designed to assess the possible factors responsible for disability status after hospital discharge in relatively healthy patients with severe pneumonia and ARDS status. Therefore, this study prospectively evaluated the possible clinical factors and biomarkers of predicting ICU mortality and 6-month outcomes in relatively healthy patients with severe pneumonia and ARDS.

\section{Patients and Methods}

2.1. Setting and Study Design. This prospective study was conducted at Kaohsiung Chang Gung Memorial Hospital, a 2,400-bed tertiary teaching hospital in southern Taiwan. The study was conducted from July 1, 2009, to June 30, 2011, for adult intubated patients (aged $\geq 18$ years) presenting with severe pneumonia and ARDS. Patients known to have tuberculosis or severe immunosuppression, such as those with human immunodeficiency virus or solid-organ or bone marrow transplantation, were excluded from the study. Furthermore, patients were excluded if they had complicated underlying diseases, were unable to take care of themselves, or had a Charlson's comorbidity index (CCI) $>5$. The study was approved by the Institutional Review Board of Chang Gung Memorial Hospital, and written informed consent was obtained from the patients or their legal representatives.

2.2. Definitions. Pneumonia was defined according to modified criteria proposed by the United States Center for Disease Control and Prevention [15]. This definition requires that two of the following criteria be satisfied: fever (increase in body temperature of $\geq 1^{\circ} \mathrm{C}$ or body temperature $>38.3^{\circ} \mathrm{C}$ ), leukocytosis $\left(25 \%\right.$ increase and leukocyte count $\geq 10,000 \mathrm{~mm}^{3}$ ) or leukopenia ( $25 \%$ decrease and leukocyte count $\leq 5,000 \mathrm{~mm}^{3}$ ), and purulent tracheal secretions ( $>25$ neutrophils per highpower field). It also requires that one of the following be satisfied: new and persistent infiltrates appearing on the chest radiograph, the same microorganisms are isolated from pleural fluid and tracheal secretions, radiographic cavitation, histological proof of pneumonia, or positive cultures from bronchoalveolar lavage $\left(\geq 1 \times 10^{4}\right.$ colony-forming units $\left./ \mathrm{mL}\right)$. This study included both community-acquired pneumonia (CAP) and hospital-acquired pneumonia (HAP); we defined HAP as pneumonia that occurred 48 hours after hospital admission [16], and all other cases were classified as CAP. ARDS status was defined as arterial oxygen tension/fraction inspired oxygen $\left(\mathrm{PaO}_{2} / \mathrm{FiO}_{2}\right)<200$ [17].

Antibiotic appropriateness was determined by considering not only antimicrobial susceptibility results [18] but also the results of an evaluation of the initial response to empirical antibiotic treatment for underlying pneumonia. After 7 days of antibiotic treatment, the clinical status of treatment was reevaluated and classified as "appropriate" if the fever had subsided, sputum production had decreased, pneumonia infiltration regression was observed on the chest radiograph, and laboratory data (including white blood cell counts, C-reactive protein levels [CRP], procalcitonin [PCT] levels, $\mathrm{PaO}_{2} / \mathrm{FiO}_{2}$ ) had improved. Additionally, if antibiotic administration was appropriate, subsequent antimicrobial susceptibility results indicated that the isolated pathogen was sensitive to the antibiotic; otherwise, the treatment was deemed "inappropriate." If clinical status could not be determined as "appropriate" or "inappropriate," or if insufficient data were available to permit evaluation of microbiological outcome, the result was designated as "indeterminate."

Patients who survived after ICU discharge were defined as "ICU survivors" and patients who died during ICU admission were defined as "ICU nonsurvivors." Patients who survived after hospital discharge were divided into two groups: life dependent and life independent. A patient was categorized as having 6-month life dependence if their Barthel's Index [19] was below or equal to 30 at the 6-month followup after hospital discharge; all others were categorized as having 6month life independence.

2.3. Data Collection and Laboratory Assays. We recorded the age, gender, pneumonia type, initial $\mathrm{PaO}_{2} / \mathrm{FiO}_{2}$ value, and CCI of every included patient. On the seventh day after ICU admission, we evaluated the antibiotic appropriateness according to the definition described above and recorded this as a clinical factor. We also prospectively calculated every patient's disease severity scores, such as the Acute Physiology and Chronic Health Evaluation II (APACHE II) score, Sequential Organ Failure Assessment (SOFA) score, and Simplified Acute Physiology Score II (SAPS II) on days 1 and 3 after ICU admission. In addition, blood samples were evaluated in each patient for several biomarkers, including CRP, PCT, D-dimer, lactate, albumin, high-mobility group protein B1 (HMGB1), interleukin-8 (IL-8), and interleukin-10 (IL-10) on days 1 and 3 after ICU admission.

For evaluating CRP, PCT, D-dimer, lactate, and albumin, these markers were measured by a real-time manner after blood sampling. CRP was measured with turbidimetric immunoassay (CRPH reagent, Synchron System Beckman, Brea, CA, USA). PCT was measured with enzyme-linked immunosorbent assay (ELISA) (VIDAS BRAHMS PCT, BioMerieux, Durham, England). D-dimer was measured with microlatex immunoturbility assay (Simens Innovance D-Dimer Reagent package insert, Dade Behring, Marburg, Germany). Lactate was measured with enzymatic method (Lactate reagent, Beckman Coulter, Brea, CA, USA). Albumin was measured with colorimetric method (Albumin reagent, KANTO TAIWAN Corporation, Taipei, Taiwan). For evaluating HMGB1, IL-8 and IL-10 and serum and plasma samples were centrifuged at $1000 \mathrm{~g}$ for 15 minutes at $4^{\circ} \mathrm{C}$ within one hour following collection. Samples were immediately stored in $1.5 \mathrm{cc}$ microcentrifuge tube at $-80^{\circ} \mathrm{C}$ until analysis for HMGB1, IL-8, and IL-10. HMGB1, IL-8, and IL-10 were measured with a commercially available ELISA (Quantikine ELISA kit, R\&D Systems, Minneapolis, MN, 
USA), and the frozen samples were preserved within 30 days before ELISA measurement for HMGB1, IL-8, and IL-10.

All clinical factors, serum biomarkers, and the day 3 : day 1 ratios of biomarker concentration or score values were analyzed as potential predictive factors of ICU mortality and 6-month outcomes. Furthermore, the length of the hospital stay, length of ICU stay, and number of mechanical ventilation (MV) days were also recorded as factors to predict 6-month outcomes after hospital discharge.

2.4. Statistical Analysis. Categorical variables were analyzed using the Chi-squared test or Fisher's exact test where appropriate, and continuous variables were compared using the Student's $t$-test or the Mann-Whitney $U$ test. Multivariate logistic regression analysis was performed to identify independent clinical factors and biomarkers of ICU mortality and 6-month outcome, respectively. All variables considered as risk factors with a $P$ value $<0.10$ by univariate analysis were entered into the multivariate model. If individual variables had a $P$ value $<0.05$ in the multivariate model, a backward elimination procedure was used to identify the final independent risk factors.

Results are presented as absolute numbers (percentage) or mean and standard deviation (SD). Adjusted odds ratios (AORs) and 95\% confidence intervals (CIs) were reported for the logistic regression analysis. A 2 -tailed $P$ value $<0.05$ was considered significant. All statistical analyses were performed using the Statistical Package for the Social Sciences (SPSS) 14.0 software package (SPSS Inc., Chicago, IL, USA).

\section{Results}

In total, 342 patients with severe pneumonia and ARDS admitted to medical ICUs within the 2-year period from July 1,2009 , to June 30,2011 , and only 79 relatively healthy patients met the inclusion criteria. Twenty-three patients refused blood tests due to being in critical condition; therefore, only 56 patients were enrolled in our study. Among the 56 patients, 40 patients were ICU survivors and 16 were ICU nonsurvivors, resulting in a mortality rate of $28.6 \%$. Only 35 patients were successfully discharged from the hospital, and 25 patients were considered to be 6-month life independent by Barthel's Index criteria (Figure 1).

\subsection{Characteristics of ICU Survivors and Nonsurvivors.} Table 1 shows the clinical factor characteristics between the ICU survivors and nonsurvivors. We found no statistical intergroup differences in gender, pneumonia type, initial $\mathrm{PaO}_{2} / \mathrm{FiO}_{2}$ value, $\mathrm{CCI}$, and the ratios of the SOFA scores on day 3 to those on day 1 . However, we also determined that initial adequate antibiotic use, low physiologic scores for APACHE II, SOFA, and SAPS II at days 1 and 3 , and a large day 3: day 1 value for the APACHE II and SAPS II scores may result in a low ICU mortality rate in patients with severe pneumonia and ARDS.

Table 2 indicates the serum biomarker characteristics between ICU survivors and nonsurvivors. We noted low day
3 levels of CRP and lactate, low day $1 \mathrm{D}$-dimer level, and low day 1 and day 3 levels of HMGB1 and IL-8 in ICU survivors.

\subsection{Characteristics of 6-Month Life-Dependent and 6-Month} Life-Independent Patients. Table 3 describes the clinical factor characteristics between 6-month life-independent and life-dependent patients. We found that old age, HAP, high CCI value, the ratio of the SOFA scores on day 3 to those on day 1, long ICU or hospital stay, and a high number of MV days may result in 6-month life dependence after hospital discharge.

Table 4 shows the serum biomarker characteristics between 6-month life-independent and life-dependent patients. We determined that low albumin level at day 1 and the day 3: day 1 ratio for the albumin level, high HMGB1 level at day 1 and day 3, and high lactate level at day 3 may be associated with 6-month life dependence after hospital discharge.

3.3. Predictors for ICU Mortality and 6-Month Life Dependence. In this study, we used the multivariate logistic regression method to determine independent clinical factors and biomarkers of predicting ICU mortality and 6-month life dependence, respectively. We found that initial appropriate use of antibiotics $(P=0.037)$ was an independent clinical factor, and HMGB1 concentration at day 1 was an independent biomarker of ICU mortality (Table 5). Using multivariate logistic regression, we found no independent clinical predictor for 6-month life dependence; however, albumin level at day $1(P=0.049)$ was confirmed to be an independent biomarker of 6-month life dependence after hospital discharge (Table 6).

\section{Discussion}

Underlying comorbidity has been reported to be an important prognostic factor in critically ill patients and has been shown to be an important determinant of ICU outcome [20-23]. The CCI $[24,25]$ was developed to predict 1-year mortality among medical patients and is one of the most frequently used measures of comorbidity [26]. Comorbidities are often included in the exclusion criteria while designing trials to avoid their confounding influence on the study outcome. Patients with few underlying diseases tend to have good clinical outcomes even after being in critical condition; however, ICU mortality or long-term disability can result in relatively healthy patients with severe pneumonia and ARDS [13]. A previous study suggested that people with CCI scores $>5$ have essentially an $84 \%$ risk of dying at 1 year [24]; thus, we selected patients with severe pneumonia and ARDS status with CCI scores below 5 to determine the risk factors associated with ICU mortality and 6-month outcomes for clarifying the findings in "relatively healthy patients."

In this study, we assessed clinical factors such as initial appropriate antibiotic treatment, APACHE II scores, SOFA scores, SAPS II scores on days 1 and 3 after disease onset, and we found that the day 3 : day 1 ratios of the APACHE II and 


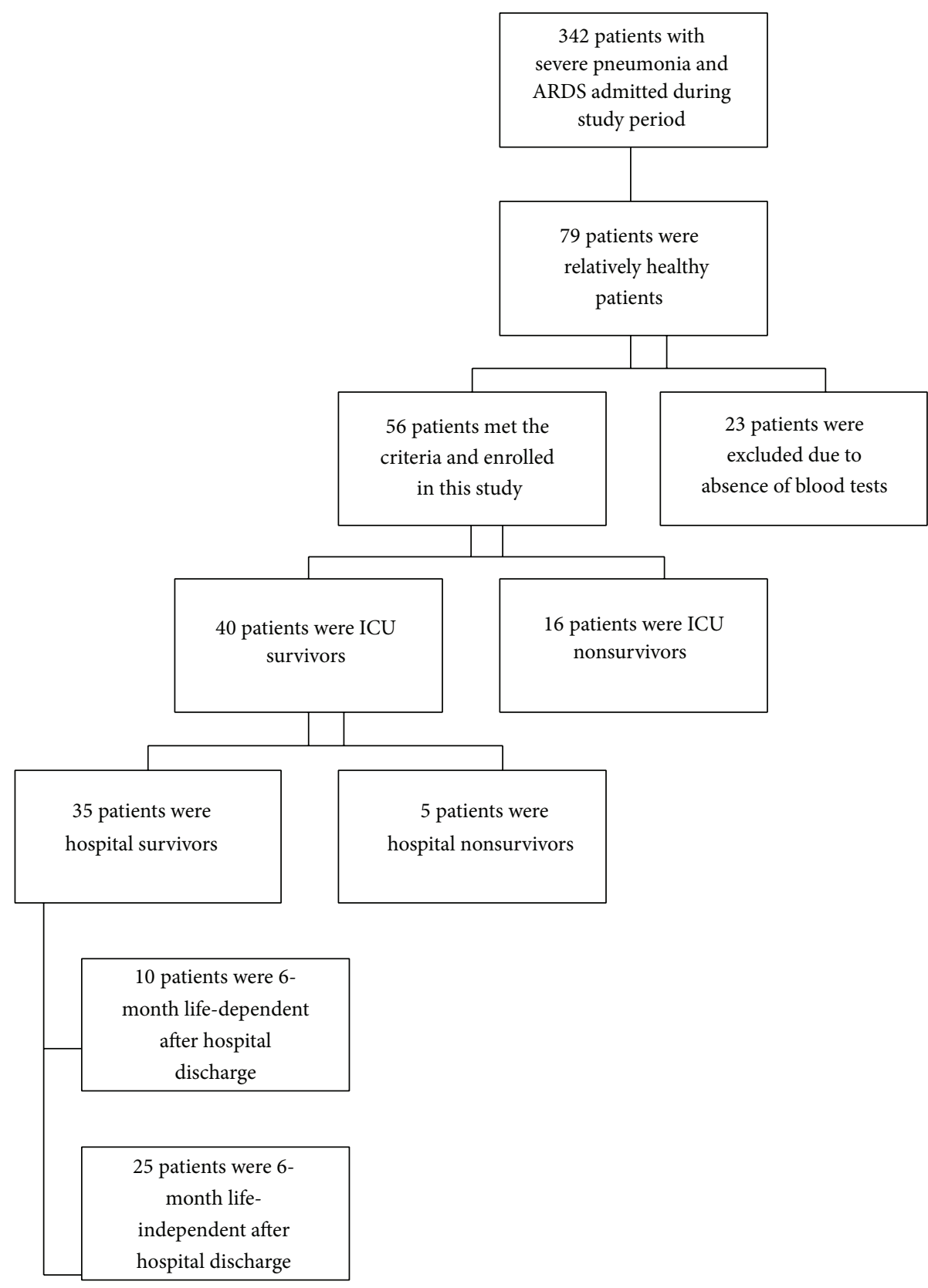

FIGURE 1: Flow chart of patient selection in this study: 342 patients with severe pneumonia and ARDS were admitted to medical ICUs within the study period, while 56 patients were enrolled in this study. Among the 56 patients, 40 patients were ICU survivors and 16 were ICU nonsurvivors. Only 35 patients were successfully discharged from the hospital, and 25 patients were considered as 6-month life independent.

SAPS II scores were associated with ICU mortality. Multivariate analysis revealed that initial appropriate use of antibiotic was an independent clinical factor for ICU mortality. A previous study demonstrated that inappropriate antibiotic treatment for pneumonia was associated with increased mortality [27]. Appropriateness of antibiotic treatment was assessed in terms of antimicrobial coverage, defined as an antimicrobial susceptibility result indicating that the isolated pathogen was sensitive to the administered antibiotic [18]. However, the relevance of pharmacokinetic-pharmacodynamic relationships in optimizing drug exposure, even if the drug is administered in a timely manner, has been progressively highlighted [28, 29]. Timely administration of an optimized dose of the right antibiotic would result in a good clinical response. Regarding this issue, careful assessment of the clinical response would be helpful in determining whether the antibiotic is appropriate. We defined "initial, appropriate use of antibiotic" according to microbiological data as well as the results of an assessment of clinical response. By using such a strict definition, we found that initial appropriate antibiotic treatment was an independent clinical predictor of ICU mortality in relatively healthy patients with severe pneumonia and ARDS. 
TABLE 1: Clinical factors characteristics between ICU survivors and nonsurvivors.

\begin{tabular}{lccc}
\hline & $\begin{array}{c}\text { ICU survivors } \\
(n=40)\end{array}$ & $\begin{array}{c}\text { ICU } \\
\text { nonsurvivors } \\
(n=16)\end{array}$ & $P$ \\
\hline Age & $63.43 \pm 13.95$ & $70.63 \pm 9.22$ & 0.063 \\
Sex, male & 23 & 8 & 0.767 \\
Pneumonia type & & & 0.262 \\
$\quad$ Community acquired & 23 & 7 & \\
$\quad$ Hospital acquired & 17 & 9 & \\
Initial PaO /FiO $_{2}$ & $163.05 \pm 60.68$ & $160.57 \pm 58.24$ & 0.889 \\
Charlson's comorbidity & $2.03 \pm 1.31$ & $2.62 \pm 1.31$ & 0.127 \\
index & & & $<0.001$ \\
Initial antibiotics & 17 & 0 & \\
Appropriate & 8 & 13 & \\
Inappropriate & 15 & 3 & \\
Indeterminate & & & \\
APACHE II score & & & \\
Day 1 & $25.47 \pm 5.29$ & $29.37 \pm 5.02$ & 0.015 \\
Day 3 & $22.45 \pm 5.90$ & $30.50 \pm 6.59$ & $<0.001$ \\
Day 3 : Day 1 & $0.88 \pm 0.11$ & $1.04 \pm 0.15$ & 0.001 \\
SOFA score & & & \\
Day 1 & & & \\
Day 3 & & & \\
Day 3 : Day 1 & $0.93 \pm 0.27 \pm 2.62$ & $11.38 \pm 2.82$ & $<0.001$ \\
SAPS II score & & $1.03 \pm 0.23$ & 0.147 \\
Day 1 & & & \\
Day 3 & & & \\
Day 3 : Day 1 & & & \\
\hline
\end{tabular}

${ }^{a}$ Continuous variables were analyzed by Student's $t$-test or Mann-Whitney $U$ test, and categorical data by Chi-square test.

${ }^{b}$ ICU: intensive care unit; APACHE II: Acute Physiology and Chronic Health Evaluation II; SOFA: Sequential Organ Failure Assessment; and SAPS II: Simplified Acute Physiology Score II.

${ }^{c}$ Variables are expressed as mean (standard deviation) and categorical data are expressed as number (percentage).

With regard to serum biomarkers predicting ICU mortality, we found that high serum levels of CRP and the day 3 lactate, day $1 \mathrm{D}$-dimer, HMGB1, and day 1 or day 3 IL-8 levels were frequently observed in ICU nonsurvivors. Furthermore, day 1 HMGB1 level was an independent biomarker of ICU mortality. Data from some animal studies showed that high HMGB1 levels are associated with severity and lethality of sepsis. HMGB1 has also been reported to be associated with shock [30] and acute lung injury [31], and blocking HMGB1 with an anti-HMGB1 antibody attenuated lung injury in animal studies $[32,33]$. In human studies, high HMGB1 level has been associated with increased mortality in subjects with severe sepsis [30]. Another study showed that HMGB1 concentration did not differ between survivors and nonsurvivors and did not predict hospital mortality in patients with severe sepsis [34]. However, these studies did not select a designated patient group such as "relatively healthy patient." HMGB1 was originally named amphoterin [35] and is secreted by
TABLE 2: Serum biomarkers characteristics between ICU survivors and nonsurvivors.

\begin{tabular}{|c|c|c|c|}
\hline & $\begin{array}{l}\text { ICU survivors } \\
\quad(n=40)\end{array}$ & $\begin{array}{c}\mathrm{ICU} \\
\text { nonsurvivors } \\
(n=16)\end{array}$ & $P$ \\
\hline \multicolumn{4}{|l|}{ CRP (mg/L) } \\
\hline Day 1 & $184.68 \pm 106.53$ & $179.71 \pm 73.53$ & 0.865 \\
\hline Day 3 & $102.77 \pm 69.01$ & $239.66 \pm 67.06$ & $<0.001$ \\
\hline Day 3 : day 1 & $0.91 \pm 2.01$ & $1.62 \pm 1.15$ & 0.184 \\
\hline \multicolumn{4}{|l|}{ PCT (ng/mL) } \\
\hline Day 1 & $8.29 \pm 8.68$ & $9.48 \pm 8.32$ & 0.639 \\
\hline Day 3 & $5.98 \pm 11.952$ & $8.37 \pm 6.38$ & 0.453 \\
\hline Day 3 : day 1 & $2.67 \pm 9.38$ & $1.05 \pm 0.48$ & 0.496 \\
\hline \multicolumn{4}{|l|}{$\mathrm{D}$-dimer $(\mathrm{mg} / \mathrm{L})$} \\
\hline Day 1 & $4.08 \pm 2.07$ & $5.58 \pm 2.87$ & 0.034 \\
\hline Day 3 & $4.07 \pm 4.12$ & $5.56 \pm 2.45$ & 0.167 \\
\hline Day 3 : day 1 & $1.28 \pm 2.13$ & $1.31 \pm 1.23$ & 0.945 \\
\hline \multicolumn{4}{|l|}{ Lactate $(\mathrm{mg} / \mathrm{dL})$} \\
\hline Day 1 & $22.21 \pm 9.67$ & $5.58 \pm 2.87$ & 0.235 \\
\hline Day 3 & $16.03 \pm 7.69$ & $32.34 \pm 27.80$ & 0.001 \\
\hline Day 3 : day 1 & $0.83 \pm 0.50$ & $1.86 \pm 3.38$ & 0.061 \\
\hline \multicolumn{4}{|l|}{ Albumin $(\mathrm{g} / \mathrm{dL})$} \\
\hline Day 1 & $2.56 \pm 0.49$ & $2.45 \pm 0.39$ & 0.416 \\
\hline Day 3 & $2.59 \pm 0.47$ & $2.36 \pm 0.35$ & 0.080 \\
\hline Day 3 : day 1 & $1.02 \pm 0.13$ & $0.97 \pm 0.11$ & 0.195 \\
\hline \multicolumn{4}{|l|}{ HMGB1 (pg/mL) } \\
\hline Day 1 & $1614.14 \pm 641.11$ & $2108.51 \pm 574.51$ & 0.025 \\
\hline Day 3 & $1548.81 \pm 650.96$ & $1952.45 \pm 763.77$ & 0.010 \\
\hline Day 3 : Day 1 & $1.02 \pm 0.39$ & $1.00 \pm 0.52$ & 0.908 \\
\hline \multicolumn{4}{|l|}{ IL-8 (pg/mL) } \\
\hline Day 1 & $56.24 \pm 38.10$ & $173.18 \pm 304.77$ & 0.019 \\
\hline Day 3 & $48.27 \pm 22.45$ & $97.19 \pm 44.29$ & $<0.001$ \\
\hline Day 3 : Day 1 & $1.12 \pm 0.79$ & $0.97 \pm 0.42$ & 0.472 \\
\hline \multicolumn{4}{|l|}{$\mathrm{IL}-10(\mathrm{pg} / \mathrm{mL})$} \\
\hline Day 1 & $40.01 \pm 35.10$ & $49.08 \pm 66.91$ & 0.509 \\
\hline Day 3 & $39.15 \pm 35.56$ & $39.27 \pm 28.05$ & 0.990 \\
\hline Day 3 : Day 1 & $1.16 \pm 0.83$ & $1.28 \pm 0.63$ & 0.616 \\
\hline
\end{tabular}

${ }^{a}$ Continuous variables were analyzed by Student's $t$-test or Mann-Whitney $U$ test, and categorical data by Chi-square test.

${ }^{b}$ ICU: intensive care unit; CRP: c-reactive protein; PCT: procalcitonin; HMGB1: high-mobility group protein B1; and IL: interleukin.

${ }^{\mathrm{c}}$ Variables are expressed as mean (standard deviation) and categorical data are expressed as number (percentage).

immune cells (e.g., macrophages, monocytes, and dendritic cells) through a leaderless secretory pathway [36]. Activated macrophages and monocytes secrete HMGB1 as a cytokine mediator of inflammation [30]. The immune response in relatively healthy patients would function better than the immune response in patients with a weakened immune system. It is reasonable to anticipate that high HMGB1 concentration may correlate with severity and mortality in relatively healthy patients who have severe pneumonia and 
TABLE 3: Clinical characteristics of 6-month life-independent and life-dependent patients.

\begin{tabular}{|c|c|c|c|}
\hline & $\begin{array}{l}\text { 6-month life } \\
\text { independence } \\
\quad(n=25)\end{array}$ & $\begin{array}{l}\text { 6-month life } \\
\text { dependence } \\
\quad(n=10)\end{array}$ & $P$ \\
\hline Age & $57.12 \pm 12.51$ & $75.30 \pm 8.07$ & $<0.001$ \\
\hline Sex, male & 16 & 5 & 0.474 \\
\hline Pneumonia type & & & 0.002 \\
\hline Community acquired & 20 & 2 & \\
\hline Hospital acquired & 5 & 8 & \\
\hline Initial $\mathrm{PaO}_{2} / \mathrm{FiO}_{2}$ & $173.13 \pm 63.84$ & $135.76 \pm 56.83$ & 0.117 \\
\hline $\begin{array}{l}\text { Charlson's Comorbidity } \\
\text { Index }\end{array}$ & $1.64 \pm 1.15$ & $3.20 \pm 1.13$ & 0.001 \\
\hline Initial antibiotics & & & 1.000 \\
\hline Appropriate & 10 & 4 & \\
\hline Inappropriate & 5 & 2 & \\
\hline Indeterminate & 10 & 4 & \\
\hline \multicolumn{4}{|l|}{ APACHE II score } \\
\hline Day 1 & $24.72 \pm 5.22$ & $25.80 \pm 1.64$ & 0.584 \\
\hline Day 3 & $21.04 \pm 4.63$ & $23.30 \pm 5.37$ & 0.221 \\
\hline Day 3 : Day 1 & $0.85 \pm 0.11$ & $0.91 \pm 0.11$ & 0.209 \\
\hline \multicolumn{4}{|l|}{ SOFA score } \\
\hline Day 1 & $8.00 \pm 2.75$ & $8.20 \pm 1.69$ & 0.833 \\
\hline Day 3 & $7.52 \pm 2.42$ & $6.80 \pm 2.10$ & 0.416 \\
\hline Day 3 : Day 1 & $0.96 \pm 0.20$ & $0.82 \pm 0.13$ & 0.044 \\
\hline \multicolumn{4}{|l|}{ SAPS II score } \\
\hline Day 1 & $39.24 \pm 7.17$ & $43.90 \pm 12.92$ & 0.181 \\
\hline Day 3 & $36.36 \pm 7.92$ & $39.60 \pm 10.29$ & 0.323 \\
\hline Day 3 : Day 1 & $0.93 \pm 0.09$ & $0.91 \pm 0.09$ & 0.759 \\
\hline ICU stay & $17.68 \pm 8.57$ & $27.00 \pm 8.56$ & 0.007 \\
\hline Hospital stay & $32.80 \pm 16.79$ & $61.20 \pm 19.20$ & $<0.001$ \\
\hline MV day & $15.24 \pm 8.04$ & $23.30 \pm 7.62$ & 0.010 \\
\hline
\end{tabular}

${ }^{a}$ Continuous variables were analyzed by Student's $t$-test or Mann-Whitney $U$ test, and categorical data by Chi-square test.

bICU: intensive care unit; APACHE II: Acute Physiology and Chronic Health Evaluation II; SOFA: Sequential Organ Failure Assessment; SAPS II: Simplified Acute Physiology Score II; MV: mechanical ventilation.

${ }^{c}$ Variables are expressed as mean (standard deviation) and categorical data are expressed as number (percentage).

ARDS. We also found that day 1 HMGB1 concentration was an independent biomarker of predicting ICU mortality.

With regard to predicting 6-month life dependence, we found that old age, having HAP, high CCI, and prolonged ICU stay, hospital stay, and MV days frequently contributed to 6-month life dependence. Serum biomarkers such as albumin, lactate, and HMGB1 on days 1 or 3 were associated with 6-month life dependence. We also determined that a low day 1 albumin level was an independent biomarker of predicting 6-month life dependence after hospital discharge. Interestingly, a previous report demonstrated that lower serum albumin concentrations could have been caused by ongoing inflammation, poor health status, and malnutrition; these factors have also been shown to be associated with low
TABLE 4: Serum biomarker characteristics between 6-month lifeindependent and life-dependent patients.

\begin{tabular}{|c|c|c|c|}
\hline & $\begin{array}{l}\text { 6-month life } \\
\text { independence } \\
\quad(n=25)\end{array}$ & $\begin{array}{l}\text { 6-month life } \\
\text { dependence } \\
\quad(n=10)\end{array}$ & $P$ \\
\hline \multicolumn{4}{|l|}{ CRP (mg/L) } \\
\hline Day 1 & $174.40 \pm 93.51$ & $205.24 \pm 151.19$ & 0.468 \\
\hline Day 3 & $98.52 \pm 70.53$ & $97.91 \pm 78.28$ & 0.982 \\
\hline Day 3 : day 1 & $1.09 \pm 2.53$ & $0.51 \pm 0.20$ & 0.480 \\
\hline \multicolumn{4}{|l|}{ PCT (ng/mL) } \\
\hline Day 1 & $8.15 \pm 9.47$ & $8.41 \pm 7.40$ & 0.939 \\
\hline Day 3 & $3.66 \pm 3.34$ & $11.32 \pm 22.53$ & 0.101 \\
\hline Day 3 : day 1 & $3.35 \pm 11.57$ & $1.97 \pm 4.44$ & 0.717 \\
\hline \multicolumn{4}{|l|}{$\mathrm{D}$-dimer $(\mathrm{mg} / \mathrm{L})$} \\
\hline Day 1 & $4.12 \pm 2.18$ & $4.06 \pm 1.19$ & 0.928 \\
\hline Day 3 & $4.57 \pm 4.99$ & $3.06 \pm 1.68$ & 0.358 \\
\hline Day 3 : day 1 & $1.52 \pm 2.67$ & $0.78 \pm 0.38$ & 0.390 \\
\hline \multicolumn{4}{|l|}{ Lactate $(\mathrm{mg} / \mathrm{dL})$} \\
\hline Day 1 & $20.01 \pm 8.37$ & $26.32 \pm 13.06$ & 0.090 \\
\hline Day 3 & $13.40 \pm 7.16$ & $19.45 \pm 7.84$ & 0.035 \\
\hline Day 3 : Day 1 & $0.80 \pm 0.60$ & $0.84 \pm 0.36$ & 0.849 \\
\hline \multicolumn{4}{|l|}{ Albumin (g/dL) } \\
\hline Day 1 & $2.76 \pm 0.39$ & $2.18 \pm 0.49$ & 0.001 \\
\hline Day 3 & $2.73 \pm 0.47$ & $2.39 \pm 0.39$ & 0.051 \\
\hline Day 3 : Day 1 & $0.99 \pm 0.13$ & $1.11 \pm 0.15$ & 0.017 \\
\hline \multicolumn{4}{|l|}{ HMGB1 (pg/mL) } \\
\hline Day 1 & $1425.36 \pm 578.39$ & $1881.06 \pm 731.29$ & 0.049 \\
\hline Day 3 & $1281.25 \pm 492.45$ & $2009.14 \pm 754.99$ & 0.002 \\
\hline Day 3 : Day 1 & $0.95 \pm 0.34$ & $1.20 \pm 0.50$ & 0.103 \\
\hline \multicolumn{4}{|l|}{ IL-8 (pg/mL) } \\
\hline Day 1 & $55.52 \pm 33.40$ & $49.27 \pm 53.66$ & 0.678 \\
\hline Day 3 & $50.80 \pm 23.57$ & $35.63 \pm 7.95$ & 0.057 \\
\hline Day 3 : Day 1 & $1.07 \pm 0.58$ & $1.38 \pm 1.28$ & 0.317 \\
\hline \multicolumn{4}{|l|}{ IL-10 (pg/mL) } \\
\hline Day 1 & $39.72 \pm 32.64$ & $34.88 \pm 42.36$ & 0.719 \\
\hline Day 3 & $43.03 \pm 36.08$ & $25.35 \pm 26.73$ & 0.171 \\
\hline Day 3: Day 1 & $1.18 \pm 0.88$ & $1.20 \pm 0.82$ & 0.941 \\
\hline
\end{tabular}

${ }^{\mathrm{a} C o n t i n u o u s ~ v a r i a b l e s ~ w e r e ~ a n a l y z e d ~ b y ~ S t u d e n t ' s ~} t$-test or Mann-Whitney $U$ test, and categorical data by Chi-square test.

${ }^{\mathrm{b}} \mathrm{CRP}$ : C-reactive protein; PCT: procalcitonin; HMGB1: high-mobility group protein B1; and IL: interleukin.

${ }^{\mathrm{c}}$ Variables are expressed as mean (standard deviation) and categorical data are expressed as number (percentage).

muscle mass or accelerated muscle loss [37]. Serum albumin concentration is also a marker of underlying diseases that are associated with muscle wasting; a previous study also revealed the association between lower albumin concentration and low muscle mass after careful adjustment for known health factors [37]. Several studies have described the ironbinding antioxidant properties of albumin [38], which is also a specific modulator of cellular glutathione and an important antioxidant [39]. Oxidative damage may play a crucial role in 
TABLE 5: Multivariate logistic regression analysis for identification of independent clinical factors and biomarkers of predicting ICU mortality.

\begin{tabular}{|c|c|c|c|}
\hline Variable & $\begin{array}{l}\text { Adjusted odds } \\
\text { ratio }\end{array}$ & $\begin{array}{c}\text { 95\% Confidence } \\
\text { interval }\end{array}$ & $P$ \\
\hline \multicolumn{4}{|c|}{ Clinical factors for predicting ICU mortality } \\
\hline Age & 1.506 & $0.807-2.810$ & 0.198 \\
\hline $\begin{array}{l}\text { Initial appropriate } \\
\text { antibiotics use }\end{array}$ & 0.032 & $0.001-0.814$ & 0.037 \\
\hline \multicolumn{4}{|l|}{ APACHE II score } \\
\hline Day 1 & 0.001 & $<0.001-90.210$ & 0.239 \\
\hline Day 3 & 1024.53 & $0.009-1.187$ & 0.244 \\
\hline Day 3 : day 1 & 8.566 & $0.003-27664.475$ & 0.602 \\
\hline \multicolumn{4}{|l|}{ SOFA score } \\
\hline Day 1 & 0.032 & $<0.001-34.761$ & 0.335 \\
\hline Day 3 & 2.756 & $0.711-10.687$ & 0.143 \\
\hline \multicolumn{4}{|l|}{ SAPS II score } \\
\hline Day 1 & 2.222 & $0.111-44.423$ & 0.601 \\
\hline Day 3 & 0.558 & $0.030-10.234$ & 0.694 \\
\hline Day 3 : Day 1 & 0.469 & $<0.001-28252.867$ & 0.893 \\
\hline \multicolumn{4}{|c|}{ Biomarkers for predicting ICU mortality } \\
\hline $\mathrm{CRP}(\mathrm{mg} / \mathrm{L})$ day 3 & 1.265 & $0.798-2.005$ & 0.317 \\
\hline D-dimer $(\mathrm{ng} / \mathrm{mL})$ day 1 & 1.211 & $0.818-1.793$ & 0.339 \\
\hline Lactate $(\mathrm{mg} / \mathrm{dL})$ ratio & 0.879 & $0.005-163.371$ & 0.879 \\
\hline Albumin $(\mathrm{g} / \mathrm{dL})$ day 3 & 1.075 & $<0.001-7.976$ & 0.261 \\
\hline \multicolumn{4}{|l|}{ HMGB1 (pg/mL) } \\
\hline Day 1 & 1.002 & $1.000-1.004$ & 0.026 \\
\hline Day 3 & 0.990 & $0.968-1.013$ & 0.384 \\
\hline \multicolumn{4}{|l|}{ IL8 (pg/mL) } \\
\hline Day 1 & 1.039 & $0.955-1.130$ & 0.737 \\
\hline Day 3 & 1.075 & $0.940-1.229$ & 0.291 \\
\hline
\end{tabular}

${ }^{a}$ ICU: intensive care unit; APACHE II: Acute Physiology and Chronic Health Evaluation II; SOFA: Sequential Organ Failure Assessment; SAPS II: Simplified Acute Physiology Score II; CRP: C-reactive protein; HMGB1: high-mobility group protein Bl; and IL: interleukin.

the decline of skeletal muscle that occurs during aging [40]. Taken together, these results and those of our study suggest that albumin may be a marker of skeletal muscle wasting and an independent factor for predicting life dependency in relatively healthy patients with severe pneumonia and ARDS.

The strength of this study was in the patient group selection; patients with severe pneumonia and ARDS that were included in our study had few underlying comorbidities and were relatively healthy patients; this group of patients has seldom been studied previously. In our study, 342 patients were admitted to medical ICUs within the study period and only 79 of these $(23.1 \%)$ were relatively healthy patients. This also shows the group of relatively healthy patients is rare. By analyzing the data that we collected in our study, we could elucidate possible independent clinical factors and biomarkers of predicting ICU mortality and 6-month life dependence in this patient group.
TABLE 6: Multivariate logistic regression analysis for identification of independent clinical factors and biomarkers of predicting 6-month life dependence.

\begin{tabular}{lccc}
\hline Variables & $\begin{array}{c}\text { Adjusted odds } \\
\text { ratio }\end{array}$ & $\begin{array}{c}\text { 95\% Confidence } \\
\text { interval }\end{array}$ & $P$ \\
\hline \multicolumn{4}{c}{ Clinical factors for predicting } \\
Age & 0.500 & $0.196 \sim 1.277$ & 0.147 \\
Pneumonia type & 0.067 & $0.001 \sim 3.803$ & 0.190 \\
Charlson's & & & \\
comorbidity index & 0.046 & $0.001 \sim 1.827$ & 0.101 \\
ICU stay & 0.083 & $0.001 \sim 5.067$ & 0.235 \\
Hospital stay & 0.861 & $0.670 \sim 1.105$ & 0.240 \\
MV day & 1.134 & $0.851 \sim 1.512$ & 0.390 \\
\hline \multicolumn{4}{c}{ Biomarkers of predicting 6-month life dependence } \\
Lactate (mg/dL) & & & \\
Day 1 & 0.909 & $0.737 \sim 1.127$ & 0.385 \\
Day 3 & 0.944 & $0.808 \sim 1.102$ & 0.465 \\
Albumin (g/dL) & & & \\
Day 1 & 18.675 & $1.018 \sim 342.677$ & 0.049 \\
Day 3 & 0.415 & $0.016 \sim 10.551$ & 0.595 \\
Day 3 : Day 1 & 0.056 & $0.000 \sim 148.527$ & 0.474 \\
HMGB1 (pg/mL) & & & \\
Day 1 & 1.003 & $0.999 \sim 1.006$ & 0.099 \\
Day 3 & 0.994 & $0.989 \sim 1.000$ & 0.068 \\
\hline
\end{tabular}

${ }^{\mathrm{a}} \mathrm{ICU}$ : intensive care unit; MV: mechanical ventilation; HMGB1: highmobility group protein $\mathrm{B} 1$.

However, there are still some limitations in our study. First, the study sample size was too small and this might result in a powerless outcome; however the reason for the small sample size may be due to patient characteristics. In other words, few relatively healthy patients with pneumonia would typically progress rapidly to ARDS requiring ICU admission. We tried our best to enroll patients that met our inclusion criteria during the 2-year period, although only 56 patients were able to join our study. Second, not every patient was able to be followed up to 1 year after hospital discharge; while every patient enrolled in this study was followed for a 6-month period, we could not provide 1-year outcome data.

\section{Conclusions}

Individuals in the general population are usually healthy with little comorbidity and seldom experience of ARDS after pneumonia occurs. However, if pneumonia indeed progresses to ARDS, they will be intubated and require MV and ICU care. This outcome may be unexpected and unfortunate for these relatively healthy patients. Importantly, we determined that initial appropriate antibiotic use (a clinical factor) and the day 1 HMGB1 concentration (a biomarker) were independent factors for predicting ICU mortality, and the day 1 albumin level was an independent biomarker of predicting 6-month life dependence in relatively healthy patients with severe pneumonia and ARDS status. 
Abbreviations

\begin{tabular}{|c|c|}
\hline ALI: & Acute lung injury \\
\hline AORs: & Adjusted odds ratios \\
\hline APACHE II: & $\begin{array}{l}\text { Acute Physiology and Chronic Health } \\
\text { Evaluation II }\end{array}$ \\
\hline ARDS: & Acute respiratory distress syndrome \\
\hline CAP: & Community-acquired pneumonia \\
\hline CCI: & Charlson's comorbidity index \\
\hline CIs: & Confidence intervals \\
\hline CRP: & C-reactive protein levels \\
\hline ELISA: & Enzyme-linked immunosorbent assay \\
\hline HAP: & Hospital-acquired pneumonia \\
\hline HMGB1: & High-mobility group protein $\mathrm{B} 1$ \\
\hline ICU: & Intensive care unit \\
\hline IL: & Interleukin \\
\hline MV: & Mechanical ventilation \\
\hline $\mathrm{PaO}_{2} / \mathrm{FiO}_{2}:$ & $\begin{array}{l}\text { Arterial oxygen tension/fraction inspired } \\
\text { oxygen }\end{array}$ \\
\hline PCT: & Procalcitonin \\
\hline QOL: & Quality of life \\
\hline SAPS: & Simplified Acute Physiology Score \\
\hline SD: & Standard deviation \\
\hline SOFA: & Sequential Organ Failure Assessment. \\
\hline
\end{tabular}

\section{Conflict of Interests}

The authors declare that there is no conflict of interests regarding the publication of this paper.

\section{Acknowledgments}

The work of this study was supported in part by a grant from the Chang Gung Memorial Hospital (CMRPG890861) to Chia-Cheng Tseng and the additional part of the funding was from the Taiwan National Science Council Grant (NSC962314-B-182A-144) to Meng-Chih Lin. The authors would like to thank all of the staff and clinicians in the intensive care unit who participated in this study for their support.

\section{References}

[1] S. Sharma, B. Maycher, and G. Eschun, "Radiological imaging in pneumonia: recent innovations," Current Opinion in Pulmonary Medicine, vol. 13, no. 3, pp. 159-169, 2007.

[2] E. Roupie, E. Lepage, M. Wysocki et al., "Prevalence, etiologies and outcome of the acute respiratory distress syndrome among hypoxemic ventilated patients," Intensive Care Medicine, vol. 25, no. 9, pp. 920-929, 1999.

[3] C. Brun-Buisson, C. Minelli, G. Bertolini et al., "Epidemiology and outcome of acute lung injury in European intensive care units: results from the ALIVE study," Intensive Care Medicine, vol. 30, no. 13, pp. 51-61, 2004.

[4] N. Petrucci and W. Iacovelli, "Ventilation with lower tidal volumes versus traditional tidal volumes in adults for acute lung injury and acute respiratory distress syndrome," Cochrane Database of Systematic Reviews, no. 3, Article ID CD003844, 2003.

[5] R. G. Brower, M. A. Matthay, A. Morris, D. Schoenfeld, B. T. Thompson, and A. Wheeler, "Ventilation with lower tidal volumes as compared with traditional tidal volumes for acute lung injury and the acute respiratory distress syndrome," The New England Journal of Medicine, vol. 342, no. 18, pp. 1301-1308, 2000.

[6] J. A. Milberg, D. R. Davis, K. P. Steinberg, and L. D. Hudson, "Improved survival of patients with acute respiratory distress syndrome (ARDS): 1983-1993," Journal of the American Medical Association, vol. 273, no. 4, pp. 306-309, 1995.

[7] P. Markowicz, M. Wolff, K. Djedaïni et al., "Multicenter prospective study of ventilator-associated pneumonia during acute respiratory distress syndrome: incidence, prognosis, and risk factors," American Journal of Respiratory and Critical Care Medicine, vol. 161, no. 6, pp. 1942-1948, 2000.

[8] R. O. Hopkins and M. S. Herridge, "Quality of life, emotional abnormalities, and cognitive dysfunction in survivors of acute lung injury/acute respiratory distress syndrome," Clinics in Chest Medicine, vol. 27, no. 4, pp. 679-689, 2006.

[9] D. M. Needham, C. R. Dennison, D. W. Dowdy et al., "Study protocol: the Improving Care of Acute Lung Injury Patients (ICAP) study," Critical Care, vol. 10, no. 1, article R9, 2005.

[10] C. M. Lee and L. D. Hudson, "Long-term outcomes after ARDS," Seminars in Respiratory and Critical Care Medicine, vol. 22, no. 3, pp. 327-336, 2001.

[11] T. A. Davidson, G. D. Rubenfeld, E. S. Caldwell, L. D. Hudson, and K. P. Steinberg, "The effect of acute respiratory distress syndrome on long-term survival," American Journal of Respiratory and Critical Care Medicine, vol. 160, no. 6, pp. 1838-1842, 1999.

[12] M. E. Wilcox and M. S. Herridge, "Long-term outcomes in patients surviving acute respiratory distress syndrome," Seminars in Respiratory and Critical Care Medicine, vol. 31, no. 1, pp. 55-65, 2010.

[13] M. S. Herridge, C. M. Tansey, A. Matté et al., "Functional disability 5 years after acute respiratory distress syndrome," The New England Journal of Medicine, vol. 364, no. 14, pp. 1293-1304, 2011.

[14] H. W. H. van Hees, W.-J. M.-J. Schellekens, M. Linkels et al., "Plasma from septic shock patients induces loss of muscle protein," Critical Care, vol. 15, article R233, 2011.

[15] J. S. Garner, W. R. Jarvis, T. G. Emori, T. C. Horan, and J. M. Hughes, "CDC Defionitions for nosocomial infections, 1988," American Journal of Infection Control, vol. 16, no. 3, pp. 128-140, 1988.

[16] "Guidelines for the management of adults with hospitalacquired, ventilator-associated, and healthcare-associated pneumonia," American Journal of Respiratory and Critical Care Medicine, vol. 171, no. 4, pp. 388-416, 2005.

[17] G. R. Bernard, A. Artigas, K. L. Brigham et al., "The AmericanEuropean Consensus Conference on ARDS: definitions, mechanisms, relevant outcomes, and clinical trial coordination," American Journal of Respiratory and Critical Care Medicine, vol. 149, no. 3, pp. 818-824, 1994.

[18] P. G. Davey and C. Marwick, "Appropriate vs. inappropriate antimicrobial therapy," Clinical Microbiology and Infection, vol. 14, supplement 3, pp. 15-21, 2008.

[19] C. Collin, D. T. Wade, S. Davies, and V. Horne, "The Barthel ADL Index: a reliability study," International Disability Studies, vol. 10, no. 2, pp. 61-63, 1988.

[20] S. Christensen, M. B. Johansen, C. F. Christiansen, R. Jensen, and S. Lemeshow, "Comparison of Charlson comorbidity index with SAPS and APACHE scores for prediction of mortality following intensive care," Clinical Epidemiology, vol. 3, no. 1, pp. 203-211, 2011. 
[21] D. Pittet, B. Thievent, R. P. Wenzel, N. Li, G. Gurman, and P. M. Suter, "Importance of pre-existing co-morbidities for prognosis of septicemia in critically ill patients," Intensive Care Medicine, vol. 19, no. 5, pp. 265-272, 1993.

[22] J. A. Johnston, D. P. Wagner, S. Timmons, D. Welsh, J. Tsevat, and M. L. Render, "Impact of different measures of comorbid disease on predicted mortality of intensive care unit patients," Medical Care, vol. 40, no. 10, pp. 929-940, 2002.

[23] K. M. Ho, M. Knuiman, J. Finn, and S. A. Webb, "Estimating long-term survival of critically ill patients: the PREDICT model," PLoS ONE, vol. 3, no. 9, Article ID e3226, 2008.

[24] M. E. Charlson, P. Pompei, K. A. Ales, and C. R. MacKenzie, "A new method of classifying prognostic comorbidity in longitudinal studies: development and validation," Journal of Chronic Diseases, vol. 40, no. 5, pp. 373-383, 1987.

[25] D. M. Needham, D. C. Scales, A. Laupacis, and P. J. Pronovost, "A systematic review of the Charlson comorbidity index using Canadian administrative databases: a perspective on risk adjustment in critical care research," Journal of Critical Care, vol. 20, no. 1, pp. 12-19, 2005.

[26] R. A. Deyo, D. C. Cherkin, and M. A. Ciol, "Adapting a clinical comorbidity index for use with ICD-9-CM administrative databases," Journal of Clinical Epidemiology, vol. 45, no. 6, pp. 613-619, 1992.

[27] M. S. Niederman, "Use of broad-spectrum antimicrobials for the treatment of pneumonia in seriously ill patients: maximizing clinical outcomes and minimizing selection of resistant organisms," Clinical Infectious Diseases, vol. 42, supplement 2, pp. S72-S81, 2006.

[28] F. Pea and P. Viale, "The antimicrobial therapy puzzle: could pharmacokinetic-pharmacodynamic relationships be helpful in addressing the issue of appropriate pneumonia treatment in critically ill patients?" Clinical Infectious Diseases, vol. 42, no. 12, pp. 1764-1771, 2006.

[29] F. Pea and P. Viale, "Bench-to-bedside review: appropriate antibiotic therapy in severe sepsis and septic shock-does the dose matter?" Critical Care, vol. 13, no. 3, article 214, 2009.

[30] H. Wang, O. Bloom, M. Zhang et al., "HMG-1 as a late mediator of endotoxin lethality in mice," Science, vol. 285, no. 5425, pp. 248-251, 1999.

[31] E. Abraham, J. Arcaroli, A. Carmody, H. Wang, and K. J. Tracey, "Cutting edge: HMG-1 as a mediator of acute lung inflammation," Journal of Immunology, vol. 165, no. 6, pp. 29502954, 2000.

[32] E. N. Ogawa, A. Ishizaka, S. Tasaka et al., "Contribution of highmobility group box-1 to the development of ventilator-induced lung injury," American Journal of Respiratory and Critical Care Medicine, vol. 174, no. 4, pp. 400-407, 2006.

[33] V. S. Patel, R. A. Sitapara, A. Gore et al., "High Mobility Group Box-1 mediates hyperoxia-induced impairment of Pseudomonas aeruginosa clearance and inflammatory lung injury in mice," American Journal of Respiratory Cell and Molecular Biology, vol. 48, no. 3, pp. 280-287, 2013.

[34] S. Karlsson, V. Pettilä, J. Tenhunen, R. Laru-Sompa, M. Hynninen, and E. Ruokonen, "HMGB1 as a predictor of organ dysfunction and outcome in patients with severe sepsis," Intensive Care Medicine, vol. 34, no. 6, pp. 1046-1053, 2008.

[35] J. Parkkinen, E. Raulo, J. Merenmies et al., "Amphoterin, the 30$\mathrm{kDa}$ protein in a family of HMG1-type polypeptides. Enhanced expression in transformed cells, leading edge localization, and interactions with plasminogen activation," Journal of Biological Chemistry, vol. 268, no. 26, pp. 19726-19738, 1993.
[36] J. R. Klune, R. Dhupar, J. Cardinal, T. R. Billiar, and A. Tsung, "HMGB1: endogenous danger signaling," Molecular Medicine, vol. 14, no. 7-8, pp. 476-484, 2008.

[37] M. Visser, S. B. Kritchevsky, A. B. Newman et al., "Lower serum albumin concentration and change in muscle mass: the Health, Aging and Body Composition study", American Journal of Clinical Nutrition, vol. 82, no. 3, pp. 531-537, 2005.

[38] A. Loban, R. Kime, and H. Powers, "Iron-binding antioxidant potential of plasma albumin," Clinical Science, vol. 93, no. 5, pp. 445-451, 1997.

[39] A. M. Cantin, B. Paquette, M. Richter, and P. Larivée, "Albuminmediated regulation of cellular glutathione and nuclear factor kappa B activation," American Journal of Respiratory and Critical Care Medicine, vol. 162, no. 4, pp. 1539-1546, 2000.

[40] O. Pansarasa, L. Castagna, B. Colombi, J. Vecchiet, G. Felzani, and F. Marzatico, "Age and sex differences in human skeletal muscle: role of reactive oxygen species," Free Radical Research, vol. 33, no. 3, pp. 287-293, 2000. 


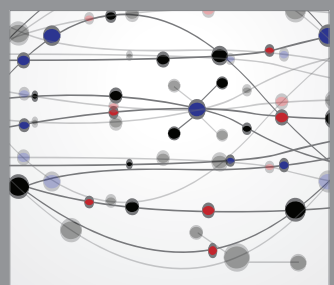

The Scientific World Journal
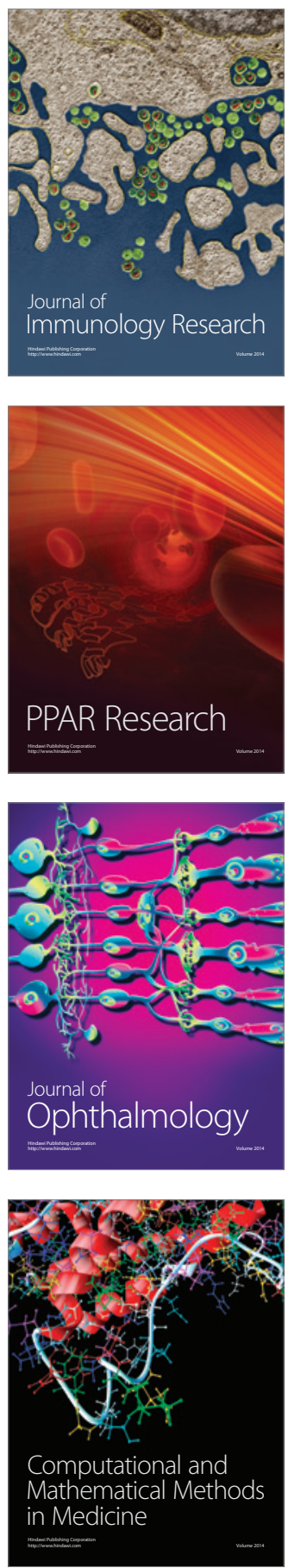

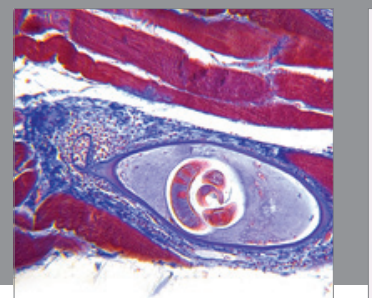

Gastroenterology

Research and Practice
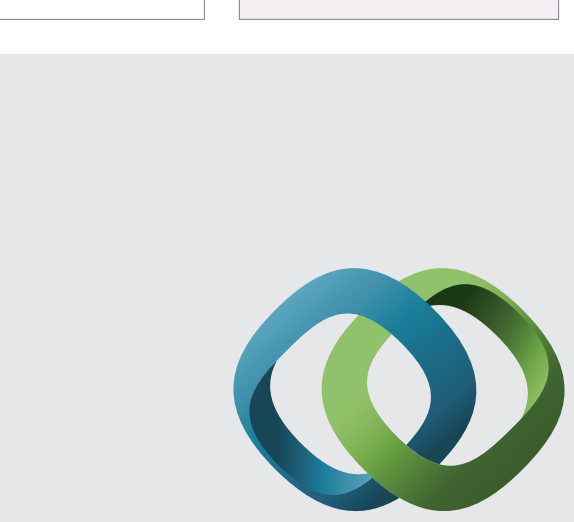

\section{Hindawi}

Submit your manuscripts at

http://www.hindawi.com
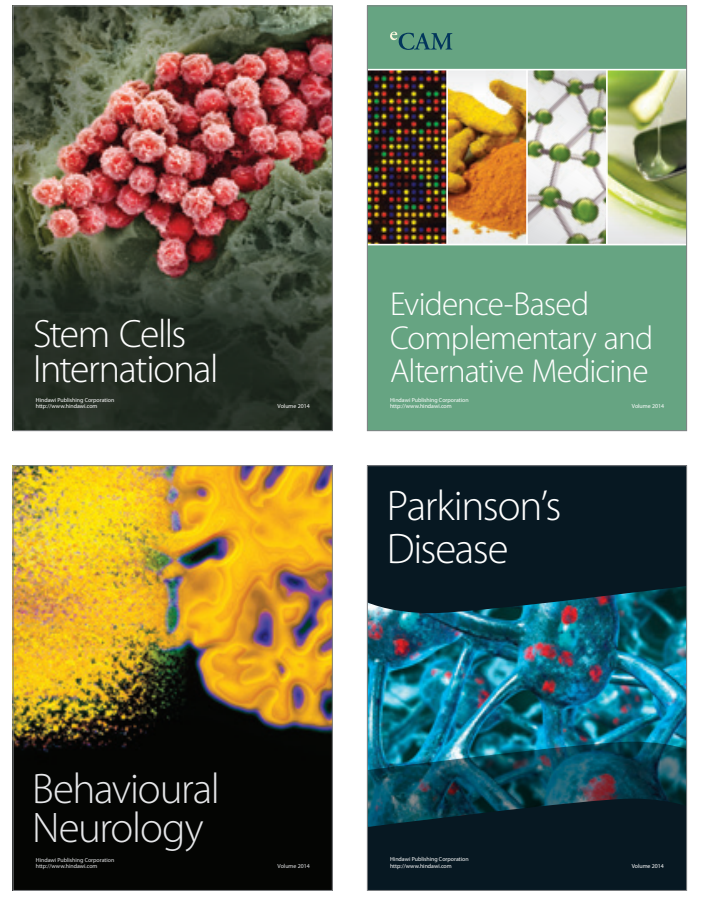
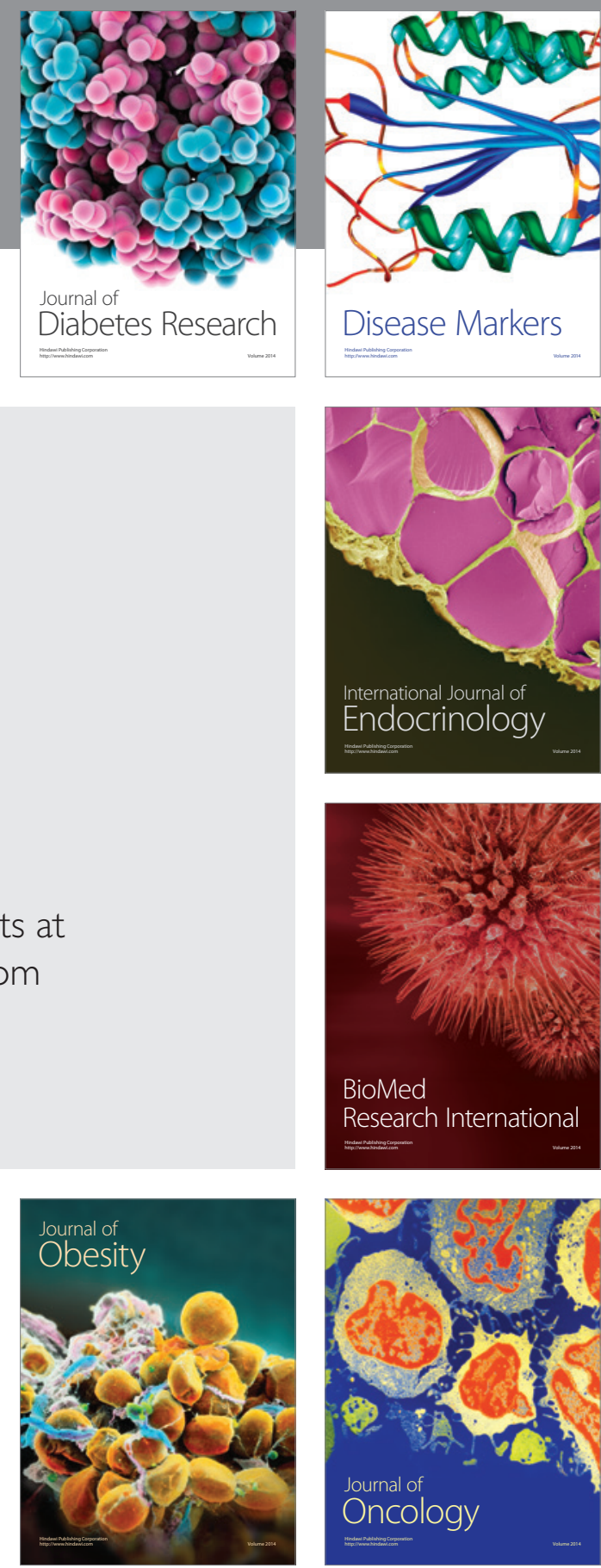

Disease Markers
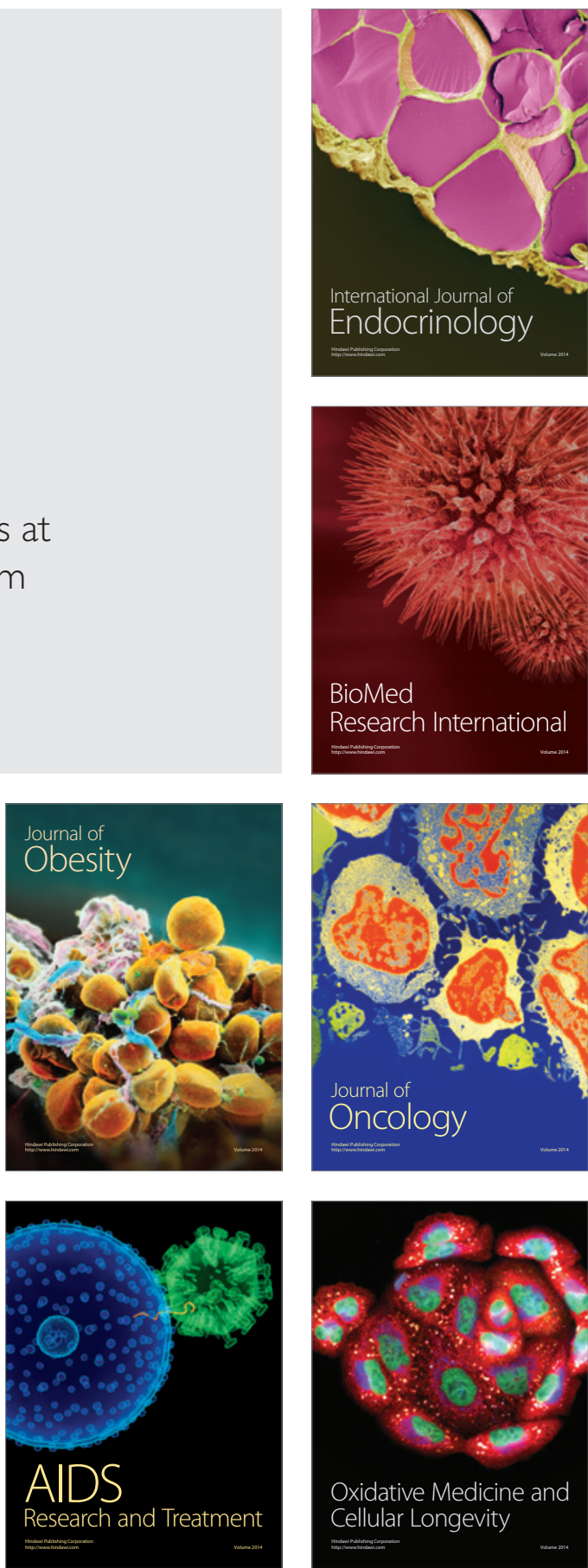